\title{
Eddy current effects on a clinical 7T-68 cm bore scanner
}

\author{
Nils Kickler • Wietske van der Zwaag • Ralf Mekle • \\ Tobias Kober • Jose P. Marques • Gunnar Krueger • \\ Rolf Gruetter
}

Received: 26 April 2009 / Revised: 18 November 2009 / Accepted: 19 November 2009 / Published online: 9 December 2009

(C) ESMRMB 2009

\begin{abstract}
Introduction Eddy currents induced by switching of magnetic field gradients can lead to distortions in short echo-time spectroscopy or diffusion weighted imaging. In small bore magnets, such as human head-only systems, minimization of eddy current effects is more demanding because of the proximity of the gradient coil to conducting structures.

Methods In the present study, the eddy current behavior achievable on a recently installed 7 tesla $-68 \mathrm{~cm}$ bore headonly magnet was characterized.

Results Residual effects after compensation were shown to be on the same order of magnitude as those measured on two whole body systems ( 3 and $4.7 \mathrm{~T}$ ), while using two to three fold increased gradient slewrates.
\end{abstract}

Keywords 7 Tesla $\cdot$ Head-only magnet $\cdot$ Eddy currents

N. Kickler · W. van der Zwaag · R. Mekle · T. Kober .

J. P. Marques · R. Gruetter

Laboratory for Functional and Metabolic Imaging,

Ecole Polytechnique Fédérale de Lausanne,

Lausanne, Switzerland

N. Kickler · W. van der Zwaag · R. Mekle $\cdot$ J. P. Marques

Department of Radiology, University of Lausanne,

Lausanne, Switzerland

T. Kober · G. Krueger

Advanced Clinical Imaging Technology, Siemens Medical

Solutions-CIBM, Lausanne, Switzerland

R. Gruetter

Departments of Radiology, Universities of Lausanne and Geneva, Lausanne, Switzerland

N. Kickler $(\varangle)$

Swiss Federal Institute of Technology Lausanne (EPFL),

SB - LIFMET, CH F0 626, Station 6, 1015 Lausanne, Switzerland

e-mail: NilsKickler@googlemail.com

\section{Introduction}

Switching of magnetic field gradients induces, via time variation of the magnetic field, eddy currents (EC) in the various conducting structures of the magnet. In turn, these currents are the origin of field variations that may persist up to several seconds after the primary gradient has been switched off. A large part of these field variations can be described by a gradient present in the same direction as the primary gradient and a $\mathrm{B}_{0}$-field shift. Reduction of eddy current effects is especially important for short echo-time spectroscopy [1] or diffusion-weighted imaging (DWI), where strong field-gradients are rapidly switched and when, as in the case of DWI, particularly sensitive readout methods, such as echo planar imaging (EPI), are commonly used [2].

Reduction of eddy currents can be achieved by active shielding of the gradient coil and by magnet design, e.g. increasing the magnet bore size with respect to the gradient coil dimension [3]. Residual gradients from eddy currents are suppressed by applying correction (or preemphasis-) currents in the respective gradient coil. Spatially homogeneous field-shifts have traditionally been minimized using a $\mathrm{B}_{0}$-shim coil and, more recently, by matching the demodulation frequency of the scanner to the shifted $\mathrm{B}_{0}$-field (information obtained from manufacturer). In small bore systems, proximity of conducting structures (such as the heat shield) to the gradient coil can lead to large eddy current effects when compared to whole body magnets, placing thereby higher demands on magnet and gradient coil design. We have recently installed an actively shielded 7 tesla scanner with a bore size of $68 \mathrm{~cm}$, equipped with an actively shielded head gradient coil. The aim of this study was to determine to what extend eddy current fields can be minimized on such a system using the vendor-supplied hardware and careful adjustment of the preemphasis 
compensation parameters using a localized method as described by Terpstra et al. [4].

\section{Materials and methods}

The study was carried out on an actively shielded 7T magnet (Magnex Scientific, Oxford, United Kingdom) with a room temperature bore of $68 \mathrm{~cm}$ diameter. The system design allowed for a cryostat length of only $2.2 \mathrm{~m}$. The 2 tesla limit of the main magnetic field remains within the cryostat and the 5 Gauss line within a distance of $4.5 \mathrm{~m}$ (radial) to about $6.5 \mathrm{~m}$ (axial) from the isocenter. The head gradient coil integrated into the system is a recent design [5] allowing for maximum gradient strengths of $80 \mathrm{mT} / \mathrm{m}$ switched at slewrates of up to $700 \mathrm{mT} /(\mathrm{m} * \mathrm{~ms})$ with second order shim fields up to $14 \mathrm{mT} / \mathrm{m}^{2}$. In this study, eddy currents from a gradient switched at $333 \mathrm{mT} /(\mathrm{m} * \mathrm{~ms})$, i.e., at about $48 \%$ of the maximum slewrate, were characterized.

Eddy current measurements and adjustment of new preemphasis parameters were performed in the magnet isocenter using an oil-filled spherical phantom (Siemens, Erlangen, Germany). A head volume coil resonator (InVivo, Orlando, USA) was used for rf- transmission and reception. The sequence used for eddy current measurement is described in detail by Terpstra et al. [4]. Briefly, at a variable delay $\tau$ after the end of a user defined test-gradient, a bar (side length $2 \mathrm{~cm}$ ) was selected within the phantom using a STEAM sequence $(\mathrm{TE}=10 \mathrm{~ms}, \mathrm{TR}=6.1$ or $6.2 \mathrm{~s}$, $\mathrm{TM}=1 \mathrm{~s}$ ). Signal was acquired in the presence of a read gradient, providing a representation of the sample profile along the bar after Fourier transform (FT). The direction of the test-gradient coincided with the readout direction. Therefore, eddy currents, caused by the test-gradient modify the phase distribution along this profile. A complex division of two acquisitions performed once with and once without application of the test-gradient retains only eddy current effects as a consequence of the test-gradient. Fitting of the phase evolution along the profile to 0 th and 1st order (offset and linear evolution in space) was used to calculate $\mathrm{B}_{0}$-shift and eddy current gradient, respectively, present at a delay $\tau$ after the end of the test-gradient. Higher order field variations were found negligible relative to the 0 th and 1 st order terms.

Eddy current gradient and $\mathrm{B}_{0}$-shifts were measured at 36 different time delays ranging from $1 \mathrm{~ms}$ to $3 \mathrm{~s}$ after the end of a $10.2-\mathrm{mT} / \mathrm{m}$ test-gradient of $2 \mathrm{~s}$ duration. This test- gradient amplitude corresponds to about $13 \%$ of the strength maximal available on the system. According to our experience with the method used here, EC correction optimized for such a gradient strength leads to an acceptable compensation for the range of gradient configurations employed by many methods. Because of the long duration of the test-gradient, eddy currents induced by its rising edge were considered neg- ligible at the time of application of the first rf pulse of the STEAM coherence generation. After complex division, zerofilling and FT, the resulting phase evolution was unwrapped and fitted in an area of $\pm 2.4 \mathrm{~cm}$ at the isocenter to determine eddy current gradient and $\mathrm{B}_{0}$-shift.

In a first step, measurements were performed with the eddy current compensation disabled (correction amplitudes and time constants were set to zero). These measurements were then used to determine new parameters for the system's preemphasis unit. The temporal decay of eddy current gradient and $\mathrm{B}_{0}$-shift values after the end of the test-gradient were fitted to a sum of exponentially decaying functions ( $\sum_{i=1}^{N} A_{i} e^{-t / \tau_{i}}$, where $N$, number of exponentials; $A_{i}$, amplitude of one of the $N$ exponentially decaying components fitted to either the eddy current gradient or the $\mathrm{B}_{0}$-shift evolution; $\tau_{i}$, decay time constant). The number of exponentials $(N)$ was successively increased until a fit residual near or below $2 \mathrm{~Hz} / \mathrm{cm}$ for the gradient and near or below $2 \mathrm{~Hz}$ for the $\mathrm{B}_{0}$-shift at all times was obtained. Adjacent decay time constants were chosen at least three-fold different $\left(\tau_{\mathrm{i}+1} / \tau_{\mathrm{i}} \geq 3\right)$ wherever possible (exception: $y$-axis, $\left(\tau_{\text {slow }} / \tau_{\text {medium }} \geq 2.6\right)$.

The time constants determined as described previously were entered directly into preemphasis unit of the scanner. The amplitudes of the exponential decays were determined iteratively as described by Terpstra et al. [4] i.e., no further calibration of the preemphasis unit was performed. In this process, the amplitude corresponding to the time constant $\tau_{\mathrm{i}}$ ( $i=1,2$ or 3 ) was adjusted by minimizing eddy current effects occurring at times longer than $5 * \tau_{\mathrm{i}-1}$ after the end of the test-gradient. Adjustments started with the amplitude corresponding to the longest time constant. Measurements of $\mathrm{B}_{0}$-shift and residual gradients were repeated after the adjustment had been performed on all axes.

To examine the influence of residual eddy currents on peakshapes in a spectroscopy experiment, a water signal was acquired at an echo- time of $9.5 \mathrm{~ms}$ from a $2 \times 2 \times 2 \mathrm{~cm}^{3}$ volume (TR $=4 \mathrm{~s}$, bandwidth $=1 \mathrm{kHz}, 4$ scans $)$ using a spherical, well- shimmed water phantom. Furthermore, in vivo acquisitions were performed with an echo-time of $6 \mathrm{~ms}$ on a volume of $2 \times 2 \times 2 \mathrm{~cm}^{3}$ situated in the occipital lobe of a human subject [6] (TR $=4 \mathrm{~s}$, bandwidth $=4 \mathrm{kHz}$, 64 scans). All spectroscopy acquisitions used the SPECIAL sequence [1] preceded by 1 st and 2 nd order shimming with FASTMAP [7]. In vivo, a home-built, unshielded, quadrature surface coil was used for signal transmission and reception. Water was suppressed using VAPOR [8]. Postprocessing of in vivo acquisitions consisted of two-times zero-filling, $2 \mathrm{~Hz}$ Gaussian weighting of the FID, Fourier transform and zero order phase correction. Human study conditions were approved by the local ethics committee.

For further comparison, $\mathrm{B}_{0}$-shifts and eddy current gradients were measured on a $3 \mathrm{~T}$ whole body scanner (Trio a Tim system, Siemens, Erlangen, Germany) with the pulse 
sequence and similar experimental setup as described previously while keeping the standard preemphasis settings (i.e., no further adjustments performed). The test-gradient as produced by the body gradient coil, was switched at $170 \mathrm{mT} /(\mathrm{m}$ $*$ ms), which was the maximum slewrate available.

\section{Results}

The decays of $\mathrm{B}_{0}$-shift and eddy current gradient on the uncompensated 7T system showed multi-exponential behavior on all axes (Fig. 1). Fit of these decays with two or three exponentially decaying functions was sufficient to achieve maximum (measured) residuals below $1 \mathrm{~Hz} / \mathrm{cm}$ for the eddy current gradient and below $6 \mathrm{~Hz}$ for the $\mathrm{B}_{0}$-shift at all times on all axes. As an example, eddy current fields produced by a $\mathrm{z}$-axis test-gradient are illustrated in Fig. 1a (gradient) and b ( $\mathrm{B}_{0}$-shift). On this axis $(z)$, before compensation, eddy currents led to a gradient of up to $47 \mathrm{~Hz} / \mathrm{cm}$ ( $1 \%$ of the preceding test-gradient) and a $\mathrm{B}_{0}$-shift of up to $160 \mathrm{~Hz}$. Compensation reduced these effects about 50 -fold to a maximal residual gradient of $1 \mathrm{~Hz} / \mathrm{cm}(0.02 \%$ of the applied test-gradient $)$ and a maximal $\mathrm{B}_{0}$-shift $<3 \mathrm{~Hz}$. Results for $x, y$ and $z$-axis are summarized in Table 1. In summary, compensating with a sum of two to three exponentials was sufficient to reduce eddy current effects on average 30 -fold.

Quality of the eddy current compensation was evaluated by observing the lineshape of a locally acquired water signal at an echo-time of $9.5 \mathrm{~ms}$. Linewidth after FASTMAP shimming was below $1 \mathrm{~Hz}$. Only minimal alterations of the peak-shape were present (Fig. 2) and remain below $2 \%$ of the peak amplitude within $\pm 1 \mathrm{~Hz}$ around the peak center.

Spectra acquired in vivo were thus devoid of any eddy current effects resulting in a high spectral resolution as evidenced by the myo-Inositol multiplet structure near $3.6 \mathrm{ppm}$ and by the clean baseline between choline (3.2 ppm) and creatine (3.0 ppm; Fig. 3). Average linewidth of the metabolite signals in this spectrum was about $8 \mathrm{~Hz}$ (LC model analysis [9]). The amplitude of the remaining water signal in the spectrum was strongly reduced to below the NAA peak height and without further correction, the baseline was flat.

On the 3T whole body scanner using standard preemphasis settings, residual eddy current gradients remained below $1 \mathrm{~Hz} / \mathrm{cm}(x$-axis: $0.4 \mathrm{~Hz} / \mathrm{cm}, y$-axis: $0.5 \mathrm{~Hz} / \mathrm{cm}, z$-axis: $0.9 \mathrm{~Hz} / \mathrm{cm})$ and $\mathrm{B}_{0}$-shifts below $9 \mathrm{~Hz}(x$-axis: $4 \mathrm{~Hz}, y$-axis: $1 \mathrm{~Hz}, z$-axis: $9 \mathrm{~Hz}$ ) at all measured delays following the testgradient.

\section{Discussion and conclusion}

The present study shows that on a $7 \mathrm{~T}$ scanner with $68-\mathrm{cm}$ bore, eddy current effects can be minimized below a
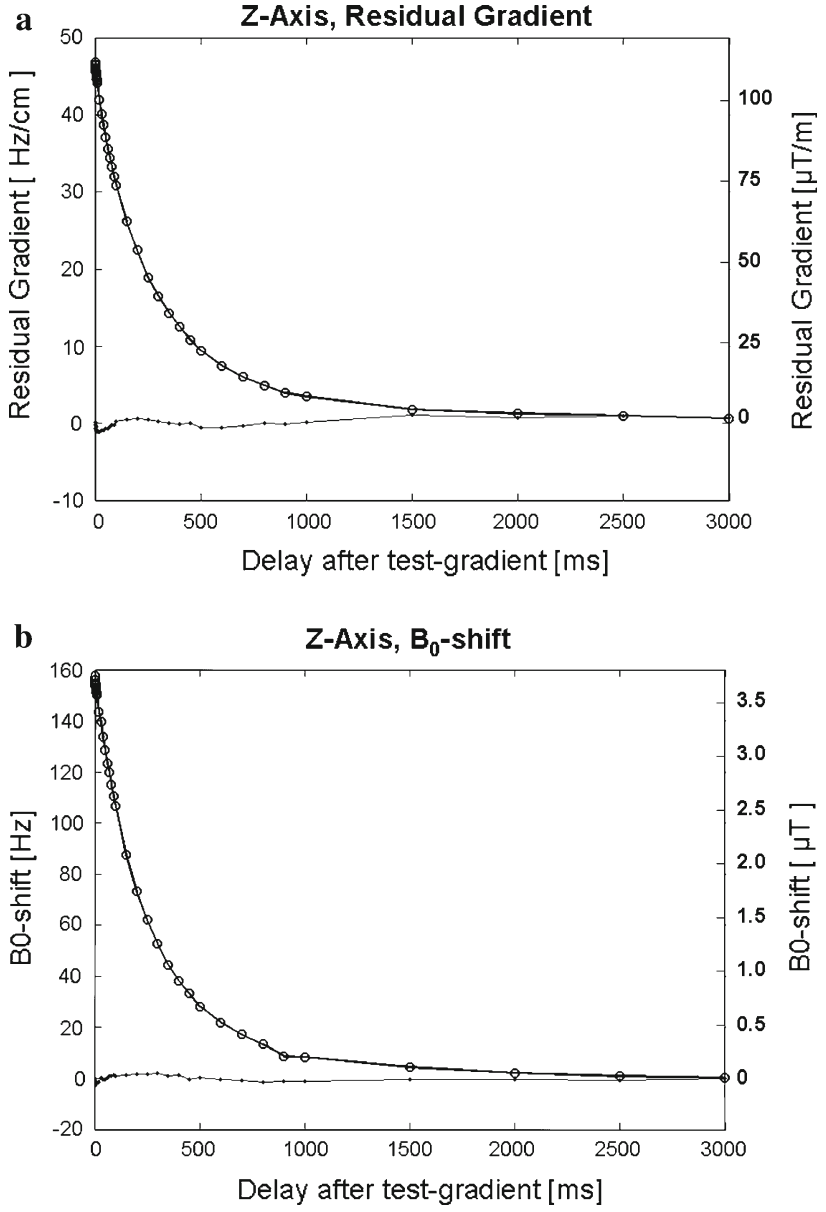

Fig. 1 Evolution of gradient (a) and $\mathrm{B}_{0}$ field-shift (b) following the application of a $z$-axis test-gradient of $10.2 \mathrm{mT} / \mathrm{m}$ amplitude and $333 \mathrm{mT} /(\mathrm{m} * \mathrm{~ms})$ slewrate. Shown are the values measured on the uncompensated system and after compensation using two time constants. Before compensation, eddy currents produced a gradient up to $47 \mathrm{~Hz} / \mathrm{cm}$ ( $1 \%$ of the amplitude of the preceding test-gradient) and a $\mathrm{B}_{0}$-shift up to $160 \mathrm{~Hz}$. Compensation reduced the gradient to near $1 \mathrm{~Hz} / \mathrm{cm}$ and the $\mathrm{B}_{0}$-shift below $3 \mathrm{~Hz}$ at all times

$\mathrm{B}_{0}$-shift of $6 \mathrm{~Hz}$ and below a residual gradient of $1 \mathrm{~Hz} / \mathrm{cm}$ following a $10.2-\mathrm{mT} / \mathrm{m}$ test-gradient commuted at a slewrate of $333 \mathrm{mT} /(\mathrm{m} * \mathrm{~ms})$. The residual $\mathrm{B}_{0}$-shift measured in the experiment remained slightly above predictions from the fit. Deviations may be caused by some compensation parameters entered iteratively into the preemphasis unit not representing exactly the amplitudes calculated $\left(A_{i}\right)$ from the fit. Nevertheless, $\mathrm{B}_{0}$-shifts and residual eddy current gradients remain almost negligible and are in size comparable to or even below values measured on a clinical 3 tesla whole body imager using standard preemphasis settings. Earlier measurements from a $4 \mathrm{~T}$ whole body system, where a maximal $\mathrm{B}_{0}$-shift of $19 \mathrm{~Hz}$ and a residual gradient near $3 \mathrm{~Hz} / \mathrm{cm} \mathrm{[4]} \mathrm{(corrected} \mathrm{for} \mathrm{the}$ test-gradient amplitude used $(13.2 \mathrm{mT} / \mathrm{m})$ ) were determined, further confirm this performance. While eddy currents on the $7 \mathrm{~T}$ were comparable in size to those measured on the 
Table 1 Time constants used for compensation of gradient and $\mathrm{B}_{0}$-shifts caused by eddy-currents. Maximum values of eddy current gradients and $\mathrm{B}_{0}$-shifts before and after compensation are indicated

\begin{tabular}{|c|c|c|c|c|c|}
\hline & \multicolumn{3}{|c|}{ Time constants (ms) } & \multirow{2}{*}{$\begin{array}{l}\text { Maximum before } \\
\text { compensation }\end{array}$} & \multirow{2}{*}{$\begin{array}{l}\text { Maximum after } \\
\text { compensation }\end{array}$} \\
\hline & Slow & Medium & Fast & & \\
\hline \multicolumn{6}{|l|}{ Gradient } \\
\hline$x$-axis & 270 & 43 & - & $30(\mathrm{~Hz} / \mathrm{cm})$ & $1(\mathrm{~Hz} / \mathrm{cm})$ \\
\hline$y$-axis & 576 & 214 & 67 & $21(\mathrm{~Hz} / \mathrm{cm})$ & $1(\mathrm{~Hz} / \mathrm{cm})$ \\
\hline$z$-axis & 458 & 120 & - & $47(\mathrm{~Hz} / \mathrm{cm})$ & $1(\mathrm{~Hz} / \mathrm{cm})$ \\
\hline \multicolumn{6}{|l|}{$\mathrm{B}_{0}$-shift } \\
\hline$x$-axis & 586 & 182 & - & $156(\mathrm{~Hz})$ & $6(\mathrm{~Hz})$ \\
\hline$y$-axis & 1121 & 318 & 104 & $86(\mathrm{~Hz})$ & $3(\mathrm{~Hz})$ \\
\hline$z$-axis & 713 & 212 & - & $160(\mathrm{~Hz})$ & $3(\mathrm{~Hz})$ \\
\hline
\end{tabular}

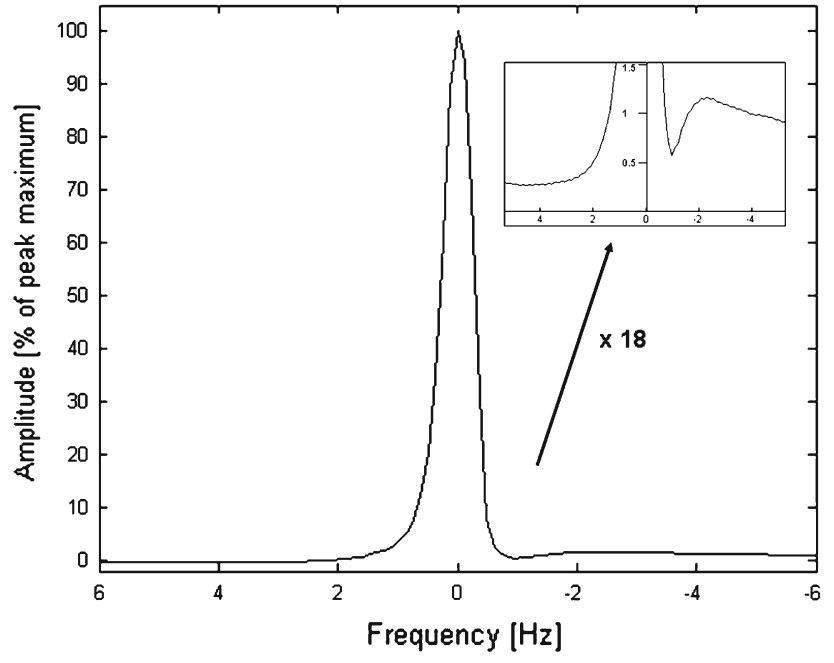

Fig. 2 Water signal acquired from a $2 \times 2 \times 2 \mathrm{~cm}^{3}$ volume in a phantom at an echo-time of $9.5 \mathrm{~ms}$ using the SPECIAL [1] sequence. The frequency width at half max of the peak was below $1 \mathrm{~Hz}$. Signal deformations caused by eddy currents remain below $2 \%$ of the peak amplitude within $\pm 1 \mathrm{~Hz}$ around the peak center

whole body magnets, the slewrate used $(333 \mathrm{mT} /(\mathrm{m} * \mathrm{~ms}))$ was nearly two to three times faster (3T: $170 \mathrm{mT} /\left(\mathrm{m}^{*} \mathrm{~ms}\right)$, 4T: $100 \mathrm{mT} /(\mathrm{m} * \mathrm{~ms}))$.

Uncompensated, decaying behavior of eddy currents was well described by not more than three exponentially decaying functions. This rather low complexity allows for highly efficient eddy current compensation by at least a factor of 20. Low eddy currents effects demonstrated on the 7T system imply that such short bore magnets can be used with little compromise on the performance with respect to eddy currents. Minimal line distortions caused by eddy currents could only be detected on a very well-shimmed signal $(<1 \mathrm{~Hz}$, Fig. 2), and remained thus below average linewidths usually observed in vivo $(8-9 \mathrm{~Hz})$. Acquiring the in vivo spectra with a different coil (surface coil) than used for preemphasis adjustment (volume coil) did not lead to reduced spec-

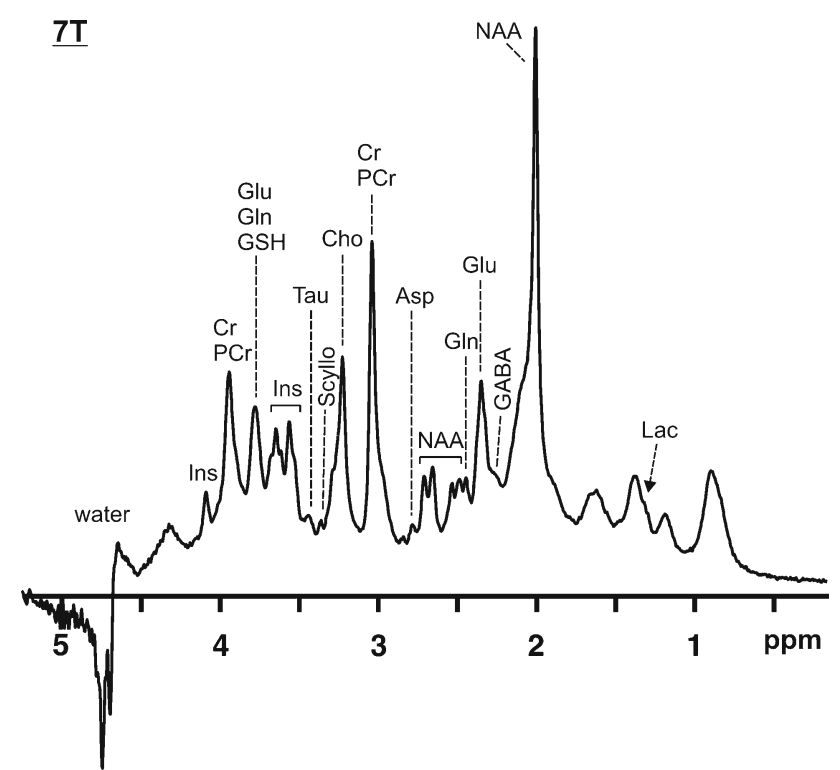

Fig. 3 Spectrum acquired at an echo-time of $6 \mathrm{~ms}$ from the occipital lobe of a healthy volunteer, using the SPECIAL sequence [6]. The average linewidth of the metabolite signals was about $8 \mathrm{~Hz}$. Postprocessing consisted of two-times zero-filling, $2 \mathrm{~Hz}$ Gaussian weighting of the FID, Fourier transform and 0th order phase correction. The spectrum is devoid of any distortions due to eddy currents and high spectral resolution is evidenced by the myo-Inositol multiplet structure near $3.6 \mathrm{ppm}$ and by the clean baseline between choline (3.2 ppm) and creatine $(3.0 \mathrm{ppm})$

tral quality. Eddy currents that might be present in conducting structures of the volume or surface coil can therefore be assumed to be negligible. Localized spectra can be acquired in vivo at very short echo times $(6 \mathrm{~ms})$ devoid of any visible deformations from eddy currents. It should be noted that, to achieve a similar spectral quality on a very small bore animal magnet, demands on residual eddy current gradients are less stringent (i.e., larger residual gradients can be accepted), as, in general, acquisitions are performed on smaller volumes than in humans [4]. 
We conclude that eddy current effects remaining on the 7T system after compensation are not performance limiting for many in-vivo applications. In terms of eddy current behavior, the $7 \mathrm{~T} / 68-\mathrm{cm}$ system can therefore be considered an interesting alternative to the installation of a whole body scanner.

Acknowledgments This work was supported by the Centre d'Imagerie BioMédicale (CIBM) of the University of Lausanne (UNIL), the Swiss Federal Institute of Technology Lausanne (EPFL), the University of Geneva (UniGe), the Centre Hospitalier Universitaire Vaudois (CHUV), the Hôpitaux Universitaires de Genève (HUG) and the Leenaards and the Jeantet Foundations.

\section{References}

1. Mlynarik V, Gambarota G, Frenkel H, Gruetter R (2006) Localized short-echo-time proton MR spectroscopy with full signal-intensity acquisition. Magn Reson Med 56(5):965-970

2. Le Bihan D, Poupon C, Amadon A, Lethimonnier F (2006) Artifacts and pitfalls in diffusion MRI. J Magn Reson Imaging 24(3):478-488
3. Chen CN, Hoult DI (1989) Biomedical magnetic resonance technology. Adam Hilger, New York

4. Terpstra M, Andersen PM, Gruetter R (1998) Localized eddy current compensation using quantitative field mapping. J Magn Reson 131(1):139-143

5. vom Endt A, Riegler J, Eberlein E, Schmitt F, Dorbert U, Krüger G, Gruetter R (2007) A high-performance head gradient coil for 7T systems. Proc Intl Soc magn Reson Med 15:451

6. Mekle R, Mlynarik V, Gambarota G, Hergt M, Krueger G, Gruetter R (2008) High-quality spectroscopy of the human brain with full signal intensity at echo times below $6 \mathrm{~ms}$ on a clinical platform at 3T and 7T. Proc Intl Soc Magn Reson Med 15:3245

7. Gruetter R (1993) Automatic, localized in vivo adjustment of all first- and second-order shim coils. Magn Reson Med 29(6):804811

8. Tkac I, Starcuk Z, Choi IY, Gruetter R (1999) In vivo 1H NMR spectroscopy of rat brain at $1 \mathrm{~ms}$ echo time. Magn Reson Med 41(4):649656

9. Provencher SW (1993) Estimation of metabolite concentrations from localized in vivo proton NMR spectra. Magn Reson Med 30(6):672-679 